\title{
Leiomyomatosis Peritonealis Disseminata: A Case Report
}

\author{
Ezzra MS*, Mokhzani WM, Zaidi Z and Siti RM \\ Department of Surgery, School of Medical Sciences, Universiti Sains Malaysia, Kelantan, Malaysia
}

${ }^{*}$ Corresponding author: Ezzra MS, Department of Surgery, School of Medical Sciences, Universiti Sains Malaysia, Kelantan, Malaysia, Tel: +604-653 3888; Fax: +604-653 6484; E-mail: ezzra128@yahoo.com

Rec Date: September 19, 2017, Acc Date: September 27, 2017, Pub Date: September 29, 2017

Citation: Ezzra MS, Mokhzani WM, Zaidi Z, Siti RM (2017) Leiomyomatosis Peritonealis Disseminata: A Case Report. Med Case Rep, Vol.3 No. $4: 44$.

\section{Abstract}

\begin{abstract}
Leiomyomatosis peritonealis disseminata (LPD) is a very rare disease characterized by formation of multiple smooth muscle tumor or nodules disseminated throughout the omental and peritoneal surfaces. It is a benign disease of unknown etiology which usually occur in women of reproductive age. The tumor may originate from the mesentery, the omentum and even the peritoneum covering the abdominal wall. Literatures have shown the association between LPD and endometriosis. Some believed the tumors arise from endometriosis foci, though no evidence to support that. The presentations are nonspecific and vague. Diagnosis is a challenge and histopathological examination (HPE) is required to diagnose LPD. Surgery is the mainstay of treatment. Prognosis is good even literatures shown that recurrence is possible after tumor resection.
\end{abstract}

Keywords: Leiomyomatosis Peritonealis Disseminate (LPD); Endometriosis; Tumor

\section{Introduction}

LPD is a rare benign disease, with an unknown etiology in women of reproductive age. LPD consists of multiple nodules adherent to and superficially invading the peritoneum, mimicking metastatic ovarian carcinoma and it occurs mainly in premenopausal women. Frozen section examination may help with the diagnosis, although the final diagnosis relies on pathological examination.

The possible causes of LPD may be divided into hormonal, subperitoneal mesenchymal stem cell metaplasia, genetic, or iatrogenic, following myoma morcellation during laparoscopic surgery.

\section{Case Presentation}

We reported a case of 56 years old lady with history of TAHBSO for multiple uterine fibroid presenting with abdominal mass for three months associated with abdominal discomfort and early satiety. On abdominal palpation revealed a firm and mobile mass measures $6 \mathrm{~cm} \times 7 \mathrm{~cm}$ in the left lumbar region. No ascites was elicited. Colonoscopy was done show normal result. Tumor markers (CEA) and LDH were normal [1]. CT imaging of the abdomen revealed multiple well defined heterogeneously enhancing interloop soft tissue lesions of varying sizes with peripheral calcifications with central necrosis. Patient then was posted for surgery.

Exploratory laparotomy, tumor debulking and omentectomy were performed. Intraoperatively, we found multiple cystic lessions varies in size largest $10 \mathrm{~cm} \times 10 \mathrm{~cm}$ arising from the omentum and mesentery. The histopathological examination reported multiple well circumscribed tumor exhibiting features of leiomyomata with degenerative changes. Postoperative period patient went without any complications. Currently patient is still under our follow up [2,3]. On the latest follow, up we found that patient having recurrence of the tumor confirmed with ultrasound findings. She now refused for any intervention as she is asymptomatic.

\section{Discussion}

LPD is a rare disease, with an unknown aetiology. LPD first discovered in 1952 by WIIlson and Peale. LPD consists of many nodules adhered to each other and superficially invading the peritoneum and it commonly seen in premenopausal women. The final diagnosis relies much on histopathological examination (HPE).

The possible etiology of LPD may be divided into hormonal, stem cell metaplasia, genetic, or iatrogenic. The finding of PR and $E R$ expression in the nodules support this hormonal theory. Literatures reported few case of LPD presented during pregnancy. One case report shows that LPD presented in postmenopausal period $[4,5]$.

Conditions where increase endogenous and exogenous female's hormones such as in pregnancy and prolonged exposure to oral contraceptive agents, has been found indicating that estrogen and progesterone play an important role in the pathogenesis of LPD. Two cases have been reported associating the disease with the use of tamoxifen for the management of breast cancer [6].

Few authors have suggested that LPD results from the implantation and proliferation of benign smooth muscle tissue or cells originating from a uterine myoma. Some abnormality 
in the $X$ chromosome and in other chromosomes $(17,12$ and 8 chromosomes) may indicate a common pathogenesis between uterine myomas and LPD. Recently, familial clustering of LPD has been reported, proposing an autosomal-dominant link with varying degrees of penetrance. Leiomyomatosis peritonealis disseminata has also been reported in men.

Preoperative diagnosis is difficult. Leiomyomatosis peritonealis disseminata usually presented with nonspecific abdominal pain or discomfort. Rarely they presented with per rectal or per vaginal bleeding. Most of the patients are asymptomatic. They also can present with symptoms such as nausea and vomiting of indigested food material.

Most patient presented late or whenever the tumor already causing compressive symptoms to adjacent organ. Radiological imagings are helpful in this situation. Several modalities such as ultrasonography, computed tomography (CT) and magnetic resonance imaging (MRI) able to detect the tumor.

The tiny nodules of disseminated peritoneal leiomyomatosis may also be below the resolution of all radiologic techniques. This imaging cannot tell the exact diagnosis. On the other hand, they may suggest the presence of a malignant condition. A spectrum of features can be seen ranging from multiple solid subcentimetre nodules like those in peritoneal carcinomatosis to large solid masses on both ultrasound and CT [7,8].

These masses may show homogeneous or heterogeneous densities with a variable enhancement pattern simillar to uterine leiomyomas. Even on magnetic resonance imaging (MRI), the masses are with a signal intensity simillar to skeletal and smooth muscle. Biopsies are required for histopathological examination and confirmation of the diagnosis. Macroscopicly these tumors are firm, round, white to grey nodules with size ranging from $0.5 \mathrm{~cm}$ to $10 \mathrm{~cm}$ in diameter.

Upon cutting the surface, they resemble uterine leiomyoma with firm, white and whorled architecture. Microscopically, the round nodules consist of mature fusiform smooth muscle cells and these cells are arranged in interdigitating fascicles $[9,10]$. The nodules are lack of mitotic figures or the mitotic index (MI) is less than $3 / 10$ high power field (HPF).

Cellular atypia, nuclear polymorphy, hyperchromasia, and tumor cell necrosis are absent in LPD while a leiomyosarcoma has a higher $\mathrm{Ml}$ and shows nuclear atypia, tumour necrosis, and infiltrative growth into adjacent structures/organs (Figures 1-3).

LPD is a benign condition. However, cytological atypia, nuclear polymorphism, hyperchromasia, tumor cell necrosis and increased mitotic figures are histological signs of malignant transformation. Some cases of malignant transformation in patients suffering from LPD have been reported and the incidence is unknown. In a review of 103 case reports, Beckers described six cases with malignant leiomyosarcoma diagnosed shortly after the diagnosis of LPD was made $[11,12]$.

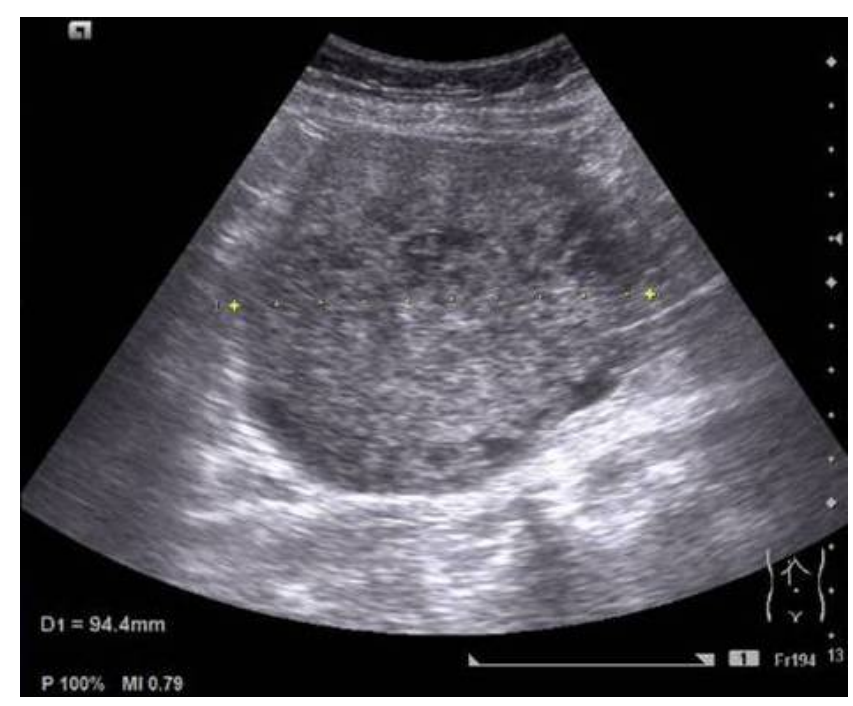

Figure 1 Ultrasound image demonstrates a well-defined lesion heterogenous with multiple hypoechoic region within suggestive of necrosis.

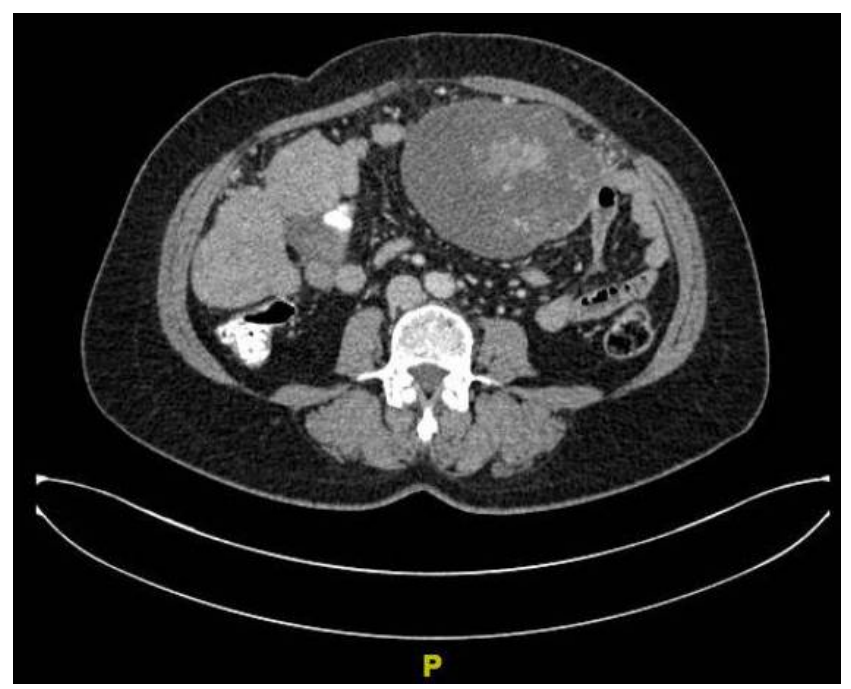

Figure 2 CECT imaging demonstrates a well-defined heterogeneously enhancing interloop soft tissue lesion peripheral calcifications with central necrosis.

The treatment of LPD can be devided into surgical and nonsurgical. Surgery is the mainstay of treatment. However, there are no firm guidelines in the literature with regard to the management of these patients. 


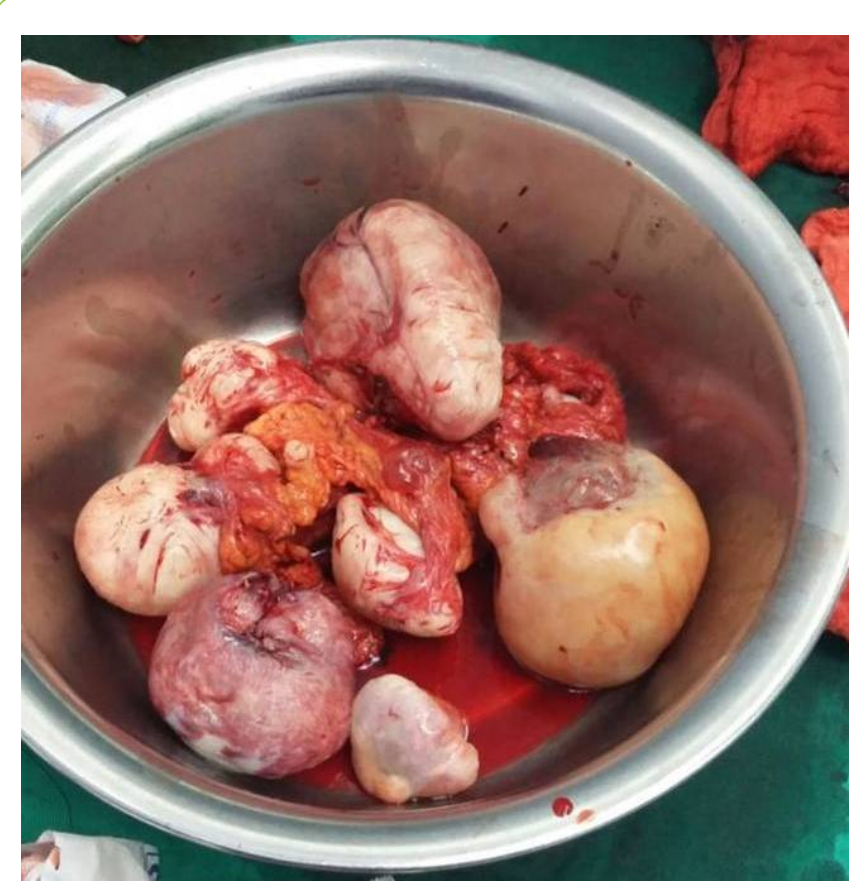

Figure 3 Leiomyomatosis peritonealis disseminata nodules resected from the mesentery and omentum of various size.

Many authors advocate a conservative approach in women with reproductive age. Reducing exposure to estrogen is sufficient to cause regression of LPD. LPD exhibits a benign biological behavior, and the decline in sex hormone levels in the body leads to regression of the disease Regression of LPD was reported with hormonal therapy using gonadotropinreleasing hormone (GnRH) agonists, megestrol acetate, and danazol. Recurrence of LPD has been reported even after a radical surgical approach surgical treatment may include exploration and debulking surgery. Recurrence of LPD has been reported even after a radical surgical approach.

\section{Conclusion}

In conclusion, LPD is very rare and difficult to diagnose. Diagnosis of LPD should be based on histopathological examination and imaging studies have a limited role. Surgery plays a big role in management of LPD. Patients should be followed carefully after surgery. This is because these tumors, though benign, could re-grow and cause symptoms or transform to malignancy.

\section{References}

1. Taubert HD, Wisser SE, Haskins AL (1965) Leiomyomatosis disseminate: An unusual complication of genital leiomyomata. Obstet Gynecol 25: 561-574.

2. Miyake T, Enomoto T, Ueda Y, Ikuma K, Morii E, et al. (2009) A case of disseminated peritoneal leiomyomatosis developing after laparoscope-assisted myomectomy. Gynecol Obstet Invest 67: 96-102.

3. Hales HA, Peterson CM, Jones KP, Quinn JD (1992) Leiomyomatosis peritonealis disseminata treated with a gonadotropin-releasing hormone agonist $A$ case report. Am J Obstet Gynecol 167: 515-516.

4. Willson Jr J, Peale AR (1952) Multiple peritoneal leiomyomas associated with a granulosa-cell tumor of the ovary. Am J Obstet Gynecol. 1952 Jul; 64(1):204-8.

5. Halama N, Grauling-Halama SA, Daboul S (2005) Familial clustering of leiomyomatosis peritonealis disseminata: An unknown genetic syndrome? BMC Gastroenterol 5: 33.

6. Heinig J, Neff A, Cirkel U, Klockenbusch W (2003) Recurrent leiomyomatosis peritonealis disseminata after hysterectomy and bilateral salpingo-oophorectomy during combined hormone replacement therapy. Eur J Obstet Gynecol Reprod Biol 111: 216-218.

7. Butnor KJ, Burchette JL, Robboy SJ (1999) Progesterone receptor activity in leiomyomatosis peritonealis disseminata. Int J Gynecol Pathol 18: 259-264.

8. Parente JT, Levy J, Chinea F, Espinosa B, Brescia MJ (1995) Adjuvant surgical and hormonal treatment of leiomyomatosis peritonealis disseminata A case report. J Reprod Med 40: 468-470.

9. Fasih N, Prasad Shanbhogue AK, Macdonald DB, Fraser-Hill MA, Papadatos D, et al. (2008) Leiomyomas beyond the uterus: An unusual location, rare manifestations. Radiographics 28: 1931-1948.

10. Singh G, Gordon-Harris L, Frazer GB, Walker SO (2002) Case report: Leiomyomatosis peritonealis disseminate-Diagnosis by sonography. IJRI 12: 497-500.

11. Cohen DT, Oliva E, Hahn PF, Fuller AF, Lee SI (2007) Uterine smooth-muscle tumors with unusual growth patterns: Imaging with pathologic correlation. AJR Am J Roentgenol 188: 246-255.

12. Yamaguchi T, Imamura $\mathrm{Y}$, Yamamoto T, Fukuda M (2003) Leiomyomatosis peritonealis disseminata with malignant change in a man. Pathol Int 53: 179-185. 